\title{
Sustained response to single agent therapy with continuous lenalidomide in a pre-treated high-risk multiple myeloma patient
}

\author{
E. Willenbacher, W. Willenbacher, E. Gunsilius \\ Myeloma Service, Department of Haematology and Oncology, Medical University of Innsbruck, Innsbruck, Austria
}

Received 3 March 2012; accepted 20 March 2012

In multiple myeloma cells, particular genomic alterations, such as del17p, are considered to be associated with higherrisk disease. However, in recent years, the introduction of thalidomide, bortezomib and lenalidomide has substantially enriched the therapeutic armamentarium for both newly diagnosed and relapsed multiple myeloma patients with poor prognosis. We describe the case of such a patient (female, 60 years of age), who refused autologous stem cell transplantation and became refractory to an induction therapy with bortezomib. Subsequently, she did not tolerate a regimen containing thalidomide and therefore was started on maintenance therapy with lenalidomide. After 4 years of treatment, the patient still is in partial remission. Our case demonstrates the long-term efficacy and good tolerability of lenalidomide monotherapy in a pre-treated high-risk multiple myeloma patient. An individually adjusted dose of lenalidomide may be a good option for patients with refractory disease who are unable to tolerate or refuse to undergo autologous stem cell transplantation.

Keywords: Multiple myeloma, high-risk, del17p, low-dose lenalidomide monotherapy.

\section{Introduction}

Multiple myeloma (MM) is a relatively rare malignant plasma cell disorder. It constitutes, however, the second most common haematologic malignancy. In the course of the disease, excessive loads of monoclonal immunoglobulins or their free light chain components result in clinical symptoms such as hypercalcaemia, renal insufficiency, anaemia or osteolytic bone lesions (CRAB) [1] and, rarely, light chain amyloidosis (AL). Accordingly, pain, fatigue and bone pain constitute the most common signs of the disease beside a variety of unspecific symptoms like early osteoporosis.

Correspondence: Eberhard Gunsilius, MD, Internal Medicine V, Haematology and Oncology, Anichstrasse 35, Innsbruck University Hospital, 6020 Innsbruck, Austria.

E-mail: eberhard.gunsilius@i-med.ac.at
Today, MM is still considered incurable despite the introduction of novel therapies, such as thalidomide, bortezomib and lenalidomide in the last decade. In patients $<65$ years of age, induction therapy comprising one of these new drugs followed by high-dose melphalan and autologous stem cell transplantation is currently the treatment of choice [2].

Here, we report the case of a patient with poor prognosis based on genetic risk factors (del17p), who was refractory to a bortezomib containing regimen and did not tolerate subsequent combination therapy with thalidomide/dexamethasone (Thal/Dex), but experienced long-lasting disease control with continuous low-dose lenalidomide monotherapy.

\section{Case report}

In October 2005, a 60-year-old female patient was admitted to the Medical University Hospital in Innsbruck. She had preexisting osteoporosis and had suffered from back pain for twelve months. During work-up by a rheumatologist, pathological serum electrophoresis results were discovered and the patient was transferred to our haematology department. Apart from pain in the spinal column and right rib area, the physical examination was uneventful. Initial laboratory tests revealed monoclonal gammopathy with IgG kappa (IgG-K) light chains and mild anaemia (Table 1). A full skeletal x-ray survey showed no bone destruction, but an MRI scan revealed osteolysis at the third right rib as well as bone-marrow infiltration. In addition, we observed multiple spotted areas ("salt and pepper") in the spine compatible with a diffuse infiltration of the vertebrae. Bone marrow histology showed increased clonal plasma cells at $50 \%$, with distinct areas of up to 90\%. Fluorescent in-situ hybridisation (FISH) revealed partial deletions of chromosomes 13 (del13q) and 17 (del17p) indicating poor prognosis [3]. Based on these findings, the patient was diagnosed with high-risk IgG kappa MM stage IA according to Durie \& Salmon [4] and stage I according to the International Staging System (ISS) [5], respectively. She refused any treatment and therefore, underwent periodic monitoring.

In June 2006, disease progression was noted with increased levels of IgG-K (M-protein, $4.38 \mathrm{~g} / \mathrm{dL}$ ) and serum free kappa light-chains (FLC-K, $1460 \mathrm{mg} / \mathrm{dL}$ ). Along with self- 
Tab. 1: Laboratory parameters at presentation

\begin{tabular}{lrll} 
Parameter & Value & $\begin{array}{l}\text { Reference } \\
\text { range }\end{array}$ & Unit \\
\hline Blood sedimentation rate at $1 \mathrm{~h}$ & 84.00 & $<10$ & $\mathrm{~mm} / \mathrm{h}$ \\
\hline M-Protein IgG kappa, lgG- & 3.10 & 0.0 & $\mathrm{~g} / \mathrm{dL}$ \\
\hline Free kappa light chains in serum & 634.00 & $3.3-19.4$ & $\mathrm{mg} / \mathrm{L}$ \\
\hline B2-microglobulin & 2.60 & $1.1-2.5$ & $\mathrm{mg} / \mathrm{L}$ \\
\hline Haemoglobin & 11.30 & $12.0-15.7$ & $\mathrm{~g} / \mathrm{dL}$ \\
\hline Serum calcium & 2.42 & $2.2-2.55$ & $\mathrm{mmol} / \mathrm{L}$ \\
\hline Serum protein (total) & 9.50 & $6.6-8.7$ & $\mathrm{~g} / \mathrm{dL}$ \\
\hline Serum creatinine & 0.79 & $0.51-0.95$ & $\mathrm{mg} / \mathrm{dL}$ \\
\hline Bence-Jones-Protein (24 h urea) & 4815.00 & 0 & $\mathrm{mg} / 24 \mathrm{~h}$ \\
\hline & & &
\end{tabular}

medication with non-steroidal anti-inflammatory drugs, the patient developed an acute renal failure (serum creatinine, $3.85 \mathrm{mg} / \mathrm{dL}$; blood urea nitrogen, $99.7 \mathrm{mg} / \mathrm{dL}$ ). Upon intravenous hydration, renal function was successfully restored and the patient finally consented to therapy. In July 2006, treatment for high-risk myeloma consisted of bortezomib $1.3 \mathrm{mg} /$ $\mathrm{m}^{2}$ on days $1,4,8,11$ plus dexamethasone $20 \mathrm{mg}$ (VD) orally on days $1,2,4,5,8,9,11$ and 12 . Additionally, multiple osteolyses were present that required vertebroplasty on the collapsed lumbar vertebra III. Ibandronic acid was given intravenously every four weeks. After the second cycle, VD treatment was terminated due to progressive disease (M-protein, $5.09 \mathrm{~g} / \mathrm{dL}$; plasma cells in a bone-marrow biopsy, $70 \%$; haemoglobin, $7.6 \mathrm{~g} / \mathrm{dL}$ ). By the end of September 2006, we initiated therapy with thalidomide $100 \mathrm{mg}$ orally per day and dexamethasone $40 \mathrm{mg}$ orally on days 1-4, 9-12, 17-20. At the same time the patient underwent a radiation therapy on the thoracic spine and the os ilium (10-11/2006) in order to alleviate pain. The Thal/Dex treatment was given over 11 months and resulted in a partial response (PR) (M-protein, $0.61 \mathrm{~g} / \mathrm{dl}$; FLC-K, $285 \mathrm{mg} / \mathrm{L}$ ) (Fig. 1). In July 2007, the patient again underwent radiotherapy for pain relief at the thoracic spine.

However, in September 2007 the treatment had to be discontinued because of painful peripheral neuropathy WHO

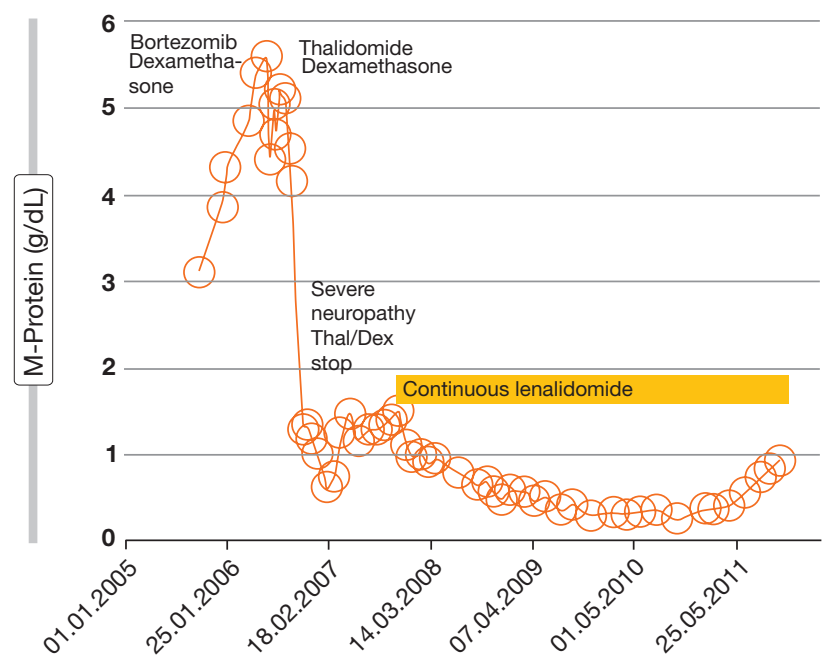

Fig. 1: M-Protein over time grade III despite reduction of the thalidomide dose. At this point, the patient once again refused autologous stem cell transplantation (ASCT) and also any further cytoreductive therapy. Due to disease progression and after intensive discussion with the patient, we initiated lenalidomide orally at $10 \mathrm{mg}$ daily in $11 / 2007$. In view of the pre-existing osteoporosis and the intention of long-term administration, no steroid was added. At this time, M-protein in serum was $1.49 \mathrm{~g} / \mathrm{dL}$ and FLC-K was $632 \mathrm{mg} / \mathrm{L}$. Renal function was normal. The new regimen led to a very good partial response (VGPR) [6]: M-protein dropped to $0.24 \mathrm{~g} / \mathrm{dL}$, FLC-K dropped to $42.8 \mathrm{mg} / \mathrm{L}$ (05/2010). After continuous therapy from 2007 till 2011, in February 2011 we decided to reduce lenalidomide dose to 5 mg daily due to the sustained effect of treatment. This resulted in a slow, gradual increase of M-protein and FLC-K. Hence, in July 2011 lenalidomide dose was elevated to $10 \mathrm{mg}$ daily because of still rising levels of M-protein $(0.83 \mathrm{~g} / \mathrm{dL})$ and FLC-K $(339 \mathrm{mg} / \mathrm{L})$. Due to further MM progression dexamethasone $24 \mathrm{mg}$ once-weekly was added in November 2011.

In the course of the long-term lenalidomide treatment, thalidomide-induced neuropathy improved gradually from WHO grade III to grade I. The patient developed low-grade leukopenia (CTC grade I) but had no other adverse events.

Almost four years after the onset of treatment with lenalidomide, the patient is still in partial remission and apart from pre-existing pain due to degenerative bone disease requiring analgesics in a good clinical state.

\section{Discussion}

Recent years have seen substantial progress towards understanding the pathogenesis of MM, including the discovery of genetic alterations in the myeloma cells with prognostic significance [7]. Deletion 17p, which was also detected in our patient, is considered to be a relatively reliable indicator of a poor prognosis. This was demonstrated by the French Myeloma Study Group which reported event-free survival of 15 months in patients with del17p versus 35 months in patients without dell7p, based on more than a thousand patients treated with conventional chemotherapy followed by transplantation. The overall survival for patients with del17p was limited to 22 months versus $75 \%$ of patients without del17p still being alive at 41 months (time of median follow-up, median not reached) [7]. Accordingly, a recently published update on risk stratification of newly diagnosed MM considers the presence of del17p a high-risk diagnostic finding [3].

For several years now, new potent drugs, such as thalidomide, bortezomib and lenalidomide, with a mode of action distinct from that of classical cytoreductive agents, have been available $[8,9]$. While the application of "old" induction regimes consisting of vincristine, doxorubicine, dexamethasone (VAD) led to a complete remission (CR) in only about $5 \%$ of patients, combination regimens including the abovementioned new drugs produced CR-rates of around 30\% [2]. The high remission rates obtained with these novel substances translate into an improved survival not only in younger but also in elderly patients not eligible for transplantation. When tolerated, however, these impressive results can be further improved by high-dose melphalan followed by ASCT.

The 60-year-old female patient described here had been diagnosed with high-risk myeloma defined by the 
presence of a double del17p and del13q in the malignant cells. Due to these high-risk features and the presence of renal impairment, she received a bortezomib-based induction regimen. Unfortunately the disease progressed during treatment and therefore, she was switched to Thal/Dex. Therapy was successful but led to a peripheral neuropathy that rendered further use of the drug impossible. In the light of the patient's anxious personality, persistence issues and the preexisting severe neuropathy, we decided on an attenuated maintenance therapy comprising lenalidomide $10 \mathrm{mg}$, daily. This is a considerably lower dose than that recommended in the Summary of Product Characteristics ( $25 \mathrm{mg}$ daily on days 1-21 of repeated 28-day cycles) [10]. In a recent study by the German Refractory Myeloma Study Group, however, a reduced lenalidomide dose (median $15 \mathrm{mg} / \mathrm{d}$ on day 1-21 of 28) combined with dexamethasone (28 mg on days 1-4, 9-12 und 17-20) demonstrated promising efficacy and safety in ten patients with relapsed and refractory MM [11]. With this regimen, the median duration of treatment was 15 months and the median event-free survival 4 months. Although our dose was even lower ( $10 \mathrm{mg}$, daily), our patient experienced a considerably longer event-free period even without dexamethasone.

Tolerability of lenalidomide was excellent over the entire treatment period. Apart from low-grade leucopenia no further toxicities were observed - not even anemia or thrombocytopenia, two of the most often reported side effects of lenalidomide [10]. In our patient, peripheral neuropathy, a recognised neurotoxic effect of thalidomide [12], was a limiting factor for this therapy. Although it is an analogue of thalidomide, the efficacy and safety profile of lenalidomide is different and includes hardly any reports of neuropathies [10].

Our patient has experienced a substantial level of disease control using continuous low-dose treatment, which has now lasted for 4 years. The good tolerability over several years makes lenalidomide a feasible option especially for sensitive or heavily pre-treated patients unable to tolerate other more stressful regimens. Beyond that, patients with relapsed and refractory MM ineligible for ASCT may benefit from low-dose lenalidomide monotherapy. Based on these observations, we think that there is a rationale for prolonged lenalidomide treatment in high-risk patients with relapsed or refractory
MM who cannot or do not want to undergo ASCT. A tailored dose of lenalidomide is well tolerated and can be given over prolonged periods.

\section{Acknowledgements}

EW acquired data and wrote the clinical part of the manuscript; WW and EG revised the data, critically reviewed the manuscript and approved the final version for submission. We thank Ulrike Posch for editorial support.

\section{Conflict of interest}

EG received financial support from Celgene Austria.

\section{References}

[1] Kyle RA, Rajkumar SV. Treatment of multiple myeloma: a comprehensive review. Clin Lymphoma Myeloma, 9: 278-88, 2009.

[2] San-Miguel JF, Mateos MV. How to treat a newly diagnosed young patient with multiple myeloma. Hematology Am Soc Hematol Educ Program, 555-65, 2009.

[3] Rajkumar SV. Multiple myeloma: 2012 update on diagnosis, riskstratification, and management. Am J Hematol, 87(1): 78-88, 2012.

[4] Durie BG, Salmon SE. A clinical staging system for multiple myeloma. Correlation of measured myeloma cell mass with presenting clinical features, response to treatment, and survival. Cancer, 36(3): 842-54, 1975.

[5] Greipp PR, San Miguel J, Durie BG, et al. International staging system for multiple myeloma. J Clin Oncol, 23(15): 3412-20, 2005.

[6] Durie BG, Harousseau JL, Miguel JS, et al. International Myeloma Working Group. International uniform response criteria for multiple myeloma. Leukemia, 20(9): 1467-73, 2006.

[7] Avet-Loiseau H, Attal M, Moreau P, et al. Genetic abnormalities and survival in multiple myeloma: the experience of the Intergroupe Francophone du Myélome. Blood, 109(8): 3489-95, 2007.

[8] Kumar SK, Rajkumar SV, Dispenzieri A, et al. Improved survival in multiple myeloma and the impact of novel therapies. Blood, 111(5): 2516-20, 2008.

[9] Laubach JP, Schlossman RL, Mitsiades CS, et al. Thalidomide, lenalidomide and bortezomib in the management of newly diagnosed multiple myeloma. Expert Rev Hematol, 4(1): 51-60, 2011.

[10] Celgene $\mathrm{GmbH}$, Revlimid: Manufacturer's summary of product characteristics. Celgene: SmPC, 2012.

[11] Schwamborn K, Gorschlüter M, Glasmacher A, et al. German Refractory Myeloma Study Group. Efficacy of dose-reduced lenalidomide in patients with refractory or recurrent multiple myeloma. Ger Med Sci, 9:Doc26, 2011.

[12] Chaudhry V, Cornblath DR, Polydefkis M, et al. Characteristics of bortezomib- and thalidomide-induced peripheral neuropathy. J Peripher Nerv Syst, 13(4): 275-82, 2008. 